\title{
Efecto de la glicerina residual en la deposición de metabolitos para la chlorella vulgaris utex 1803, mediante técnicas de inmovilización
}

Effect of the remaining glycerol in the metabolites deposition for the chlorella vulgaris utex 1803, through inmobilization techniques

\author{
Ana María Ardila-Álvarez \\ Ingeniera Química \\ Universidad Industrial de Santander \\ Bucaramanga, Colombia \\ anamariaardilaalvarez@gmail.com \\ Vanessa Edith Blanco-Suárez \\ Ingeniera Química \\ Universidad Industrial de Santander \\ Bucaramanga, Colombia \\ vannis98@hotmail.com
Andrés Fernando Barajas-Solano
Ph.D.(C) Ingeniería Química
Universidad de Santander UDES
Bucaramanga, Colombia
an.barajas@mail.udes.edu.co

\author{
Viviana Quintero-Dallos \\ M.Sc. Ingeniería Química \\ Universidad Industrial de Santander \\ Bucaramanga, Colombia \\ viviana0520@gmail.com
Susan Stepahny Villamizar-Sánchez Ingeniera Química
Universidad Industrial de Santander
Bucaramanga, Colombia
ing.susan.villamizar@gmail.com \\ Crisóstomo Barajas-Ferreira \\ M.Sc. Ingeniería Química \\ Universidad Industrial de Santander \\ Bucaramanga, Colombia \\ cbarajas@uis.edu.co
}

\begin{abstract}
Resumen- El objetivo del presente estudio fue evaluar la viabilidad del uso de glicerina como fuente de carbono, usando algas inmovilizadas para la producción de metabolitos como carbohidratos, proteínas y lípidos. Se encontró, mediante el uso de análisis estadístico, que la acumulación de hidratos de carbono se favorece con concentraciones de glicerina de $3-5 \%(\mathrm{v} / \mathrm{v})$ y 0,37 (g/L) de nitrógeno. La deposición de lípidos, se favorece con una concentración de $3 \%(\mathrm{v} / \mathrm{v})$ de glicerina y $0,04(\mathrm{~g} / \mathrm{L})$ de nitrógeno; Por otro lado, las proteínas se favorecen con mayores concentraciones de glicerina ( $5 \% \mathrm{v} / \mathrm{v})$, y una concentración de nitrógeno similar a la utilizada para hidratos de carbono.
\end{abstract}

Palabras clave- Glicerina, inmovilización, chlorella vulgaris.

\footnotetext{
Abstract- The purpose of the present study was to evaluate the viability of glycerol as carbon source using immobilized algae coupled with a modification on nitrogen $\left(\mathrm{NO}_{3}-\right)$ and phosphate $\left(\mathrm{PO}_{4}\right)$ concentration, for the production of high valuable compounds (carbohydrates, proteins and lipids). The best conditions for carbohydrate accumulation were found by statistical analysis and they were achieved within a 3-5\% (v/v) glycerol and an initial concentration of nitrogen source of $0,37(\mathrm{~g} / \mathrm{L})$. Lipid deposition was favored with a concentration of $3 \%(\mathrm{v} / \mathrm{v})$ glycerol and $0.04(\mathrm{~g} / \mathrm{L})$ nitrogen. On the other hand, proteins were favored with higher concentrations
}

of glycerol (5\% v/v) and a concentration of nitrogen similar to that used for carbohydrates

Keywords- Glycerol, inmobilization, chlorella vulgaris.

\section{INTRODUCCIÓN}

El uso de microalgas en la biotecnología se ha incrementado en los últimos años, debido a que contienen numerosos compuestos bioactivos que pueden aprovecharse para uso comercial. El tamaño de las microalgas $(<50 \mu \mathrm{m})$ implica serias dificultades a la hora de diseñar biorreactores; la pérdida de biomasa cuando se trabaja con sistemas de flujo continuo y la recolección de la biomasa celular en suspensión, es un proceso costoso y a menudo ineficaz. Con el fin de resolver ambos problemas, la biotecnología ha recurrido a técnicas de inmovilización de los organismos unicelulares fotosintéticos, los cuales se utilizan hoy en día en la industria farmacéutica, la acuicultura, la alimentación, cosmética [1], procesos de depuración [2], y la producción de biocombustibles, entre otros [3]. 
Las microalgas tienen una tendencia natural de adherirse a las superficies y crecer en ellas. Esta capacidad también se ha aprovechado con el fin de preconcentrar estas sustancias y facilitar así la medición de trazas en el medio ambiente [4]. La selección del soporte es una de las decisiones cruciales del proceso de inmovilización [5]. Los materiales de soporte deben cumplir con los siguientes criterios: ser insolubles, no biodegradables, no tóxicos, de bajo peso, flexibles, tener alta estabilidad mecánica, química y tener un bajo costo [6].

Hoy en día no hay muchas investigaciones realizadas sobre inmovilización de microalgas dedicadas a la producción de biocombustibles [7], la mayoría de los estudios están aplicados en el tratamiento de aguas residuales. Zamalloa et al. [8], diseñaron un reactor de placas paralelas para inmovilizar los nutrientes de las aguas residuales domésticas tratadas químicamente, donde se eliminó nitrógeno y fósforo con una eficiencias del $67 \%$ del nitrógeno total y $96 \%$ de fósforo total. En 2009, Hsieh, et al. (2009) construyeron un sistema para cultivar Chlorella destinada a la producción de biocombustibles con remoción de nitrógeno y fósforo simultánea [9], además de probar diferentes materiales como espuma de poliestireno, cartón, polietileno de fibra paisaje y esponja vegetal. Entre todos estos materiales, el mejor en términos de producción de biomasa y ácidos grasos fue la espuma de poliestireno [10], además de facilitar la recuperación de la biomasa.

Según Liang et al. [11], las condiciones que promueven la alta productividad y el crecimiento rápido son la suficiencia de nutrientes, es por esto que se propone un método de inmovilización de biomasa con el fin de evaluar el efecto de acumulación de biomasa y metabolitos de valor agregado.

Por otro lado, Domínguez et al [12], proponen el uso del glicerol como suplemento orgánico para el cultivo mixotrófico de la microalga, el cual se produce en cantidades significativas como subproducto de la transesterificación con una demanda comercial muy baja. Por lo tanto, el uso de glicerol constituye una propuesta innovadora y puede contribuir a mitigar efectos ambientales y diversificar la oferta en la industrial de biodiesel.

\section{METODOLOGÍA}

\section{1 Método de cultivo}

Chlorella vulgaris UTEX 1803 fue adquirida de la colección de cepas proveniente de la Universidad de Texas (Austin, Texas, EE.UU.). Inicialmente la cepa se cultivó en medio Bold Basal, cada litro de medio de cultivo está compuesto por: macronutrientes (moles) $\mathrm{NaNO}_{3}$ $\left(2,94 \times 10^{-3}\right), \mathrm{MgSO}_{4} \cdot 7 \mathrm{H}_{2} \mathrm{O}\left(3,04 \times 10^{-4}\right), \mathrm{NaCl}\left(4,28 \times 10^{-4}\right)$, $\mathrm{K}_{2} \mathrm{HPO}_{4}\left(4,31 \times 10^{-4}\right), \mathrm{KH}_{2} \mathrm{PO}_{4}\left(1,29 \times 10^{-3}\right), \mathrm{CaCl}_{2} \cdot 2 \mathrm{H}_{2} \mathrm{O}$ $\left(1,70 \times 10^{-4}\right)$ y micronutrientes (g/L) $\mathrm{ZnSO}_{4} \cdot 7 \mathrm{H}_{2} \mathrm{O}$ $\left(3,07 \times 10^{-5}\right), \mathrm{MnCl}_{2} \cdot 4 \mathrm{H}_{2} \mathrm{O}\left(7,28 \times 10^{-6}\right), \mathrm{MoO}_{3}\left(4,93 \times 10^{-6}\right)$, $\mathrm{CuSO}_{4} \cdot 5 \mathrm{H}_{2} \mathrm{O}\left(6,29 \times 10^{-6}\right), \mathrm{Co}\left(\mathrm{NO}_{3}\right)_{2} \cdot 6 \mathrm{H}_{2} \mathrm{O}\left(1,68 \times 10^{-6}\right)$, $\mathrm{H}_{3} \mathrm{BO}_{3}\left(1,85 \times 10^{-4}\right)$, EDTA $\left(1,71 \times 10^{-4}\right), \mathrm{KOH}\left(5,53 \times 10^{-4}\right)$, $\mathrm{FeSO}_{4} \cdot 7 \mathrm{H}_{2} \mathrm{O}\left(1,79 \times 10^{-5}\right)$. Se usaron reactores rectangulares de vidrio con capacidad de $2 \mathrm{~L}$, acoplando un soporte polimérico con espuma de poliuretano. El sistema reactor-polímero fue acoplado a un difusor de hule en su parte inferior, para burbujear constantemente aire suministrado desde un compresor; esto con el fin de garantizar la homogeneidad de todo el cultivo así como evitar la sedimentación celular.

\section{2 Diseño experimental}

El polímero con las células ya inmovilizadas es ahora el nuevo inóculo y el medio de cultivo se compone de glicerina residual como fuente de carbono, el medio Bold Basal con una variación de $\mathrm{NaNO}_{3} \mathrm{Como}$ fuente de nitrógeno y $\mathrm{K}_{2} \mathrm{HPO}_{4}$ como fuente de fósforo.Para determinar el efecto de la glicerina residual en la deposición de metabolitos, se planteó un diseño de experimentos factorial $3^{3}$ de composición central, tomando como variables las concentraciones de glicerina, nitrógeno y fósforo (Tabla I).

Cada experimento fue realizado por triplicado con una duración total de 16 días, en los que cada 4 días, se tomaron muestras para la cuantificación y seguimiento de la producción de biomasa, carbohidratos, lípidos, proteínas, consumo de glicerina, nitrógeno y fósforo, bajo condiciones controladas de temperatura $23 \pm 2{ }^{\circ} \mathrm{C}$ e iluminación artificial con periodos de $12 \mathrm{~h}$ día/12h noche. 
TABLA I

DISEÑO EXPERIMENTAL, CULTIVO CON MODIFICACIONES EN CONCENTRACIÓN DE GLICERINA, NITRÓGENO Y FÓSFORO

\begin{tabular}{|l|c|c|c|c|c|c|c|c|c|c|c|c|c|c|c|}
\hline Tratamiento & $\mathbf{1}$ & $\mathbf{2}$ & $\mathbf{3}$ & $\mathbf{4}$ & $\mathbf{5 *}$ & $\mathbf{6}$ & $\mathbf{7}$ & $\mathbf{8}$ & $\mathbf{9}$ & $\mathbf{1 0}$ & $\mathbf{1 1}$ & $\mathbf{1 2}$ & $\mathbf{1 3}$ & $\mathbf{1 4}$ & $\mathbf{1 5}$ \\
\hline Glicerina(\%v/v) & 1 & 1 & 5 & 5 & 3 & 1 & 1 & 5 & 5 & 3 & 0 & 6 & 3 & 3 & 3 \\
\hline $\begin{array}{l}\mathrm{KH}_{2} \mathrm{PO}_{4} \\
+\mathrm{K}_{2} \mathrm{HPO}_{4}(\mathrm{~g} / \mathrm{L})\end{array}$ & 0.12 & 0.38 & 0.12 & 0.38 & 0.25 & 0.12 & 0.38 & 0.12 & 0.38 & 0.25 & 0.25 & 0.25 & 0.04 & 0.46 & 0.25 \\
\hline $\begin{array}{l}\mathrm{NaNO}_{3} \\
(\mathrm{~g} / \mathrm{L})\end{array}$ & 0.12 & 0.37 & 0.37 & 0.12 & 0.25 & 0.37 & 0.12 & 0.12 & 0.37 & 0.25 & 0.25 & 0.25 & 0.25 & 0.25 & 0.04 \\
\hline
\end{tabular}

* El tratamiento 5 es el promedio aritmético de las pruebas 16 y 17.

Fuente: los autores.

\section{3 Consumo de glicerina}

Para la cuantificación del consumo de glicerina se filtraron las muestras de medio de cultivo, usando membranas PVDF (Di-fluoruro de polivinilideno) OlimPeak de $0.22 \mu \mathrm{m}$. La técnica empleada fue la de cromatografía líquida de alta eficacia con una columna Biorad Aminex HPX-87H acoplada a un equipo UFLC 2OAD (Shimadzu), seguida de un detector de índice de refracción RID-10A. El equipo operó a una temperatura de $65^{\circ} \mathrm{C}$, con flujo de $0.6 \mathrm{~mL} / \mathrm{min}$. La fase móvil empleada fue una solución de ácido sulfúrico $5 \mathrm{mM}$. El tiempo de cada análisis fue 30 minutos como factor de seguridad para no contaminar posteriores muestras.

\subsection{Consumo de nitrógeno}

El consumo de nitrógeno está representado por la cuantificación de Nitrato que se realizó por medio del método espectrofotométrico ultravioleta [12], la lectura de la absorbancia a una longitud de onda de 220 y $275 \mathrm{~nm}$. La corrección de los valores obtenidos debido al material orgánico presente [13], fue realizada mediante (1).

$$
\left(2 \times \lambda_{275}\right)-\lambda_{220}
$$

\subsection{Consumo de fósforo}

El consumo de fósforo está representado por la cuantificación de fosfato para lo cual se empleó el método colorimétrico del ácido Metavanadato Molibdato [13]. Se tomó una muestra de $3 \mathrm{~mL}$ de medio de cultivo, posteriormente se filtró al vacío adicionando $1 \mathrm{~mL}$ de agua destilada y $1 \mathrm{~mL}$ de ácido Metavanadato Molibdato, dejando reaccio- nar durante 10 minutos; finalmente, se realizó la lectura de la absorbancia a una longitud de onda de $750 \mathrm{~nm}$ en un espectrofotómetro (Pharo300 Merck).

\subsection{Cuantificación de biomasa}

La cuantificación de la biomasa fue evaluada utilizando el parámetro de peso seco, mediante toma de alícuotas, a partir de un raspado uniforme realizado al polímero. Las muestras se filtraron al vacío y secaron durante $24 \mathrm{~h}$ en el horno a $105{ }^{\circ} \mathrm{C}$ para posteriormente transferirlas a un desecador por $12 \mathrm{~h}$. Finalmente, por diferencia de peso del papel filtro se obtuvo las cantidades en gramos de biomasa.

\subsection{Cuantificación de metabolitos}

Los carbohidratos fueron cuantificados siguiendo el método colorimétrico fenol-ácido sulfúrico propuesto por Dubois et al. [14]. Las absorbancias se realizaron a $480 \mathrm{~nm}, 485 \mathrm{~nm}, 487 \mathrm{~nm}$ y $490 \mathrm{~nm}$, para identificar xilosa, arabinosa, glucosa, galactosa y fructosa, respectivamente.

La extracción de lípidos fue realizada siguiendo el protocolo propuesto por Chen et al. [15]. Se evaluó la absorbancia a $260 \mathrm{~nm}$ en un espectrofotómetro (Pharo300, Merck).

El proceso de cuantificación de proteínas inició con la extracción de las mismas por medio del método de Rausch (1981), empleado por Chen \& Vaidyanathan [16]. Para el cálculo de la cantidad de proteínas totales en la muestra se empleó el método de cuantificación de proteínas por reacción de Folin [17], empleado por Dorey \& Draves [18]. 


\section{RESULTADOS}

\subsection{Producción de carbohidratos}

En la Tabla II se presenta una comparación entre los tratamientos con mejores resultados en cuanto a producción de carbohidratos, siendo el tratamiento 3 el que reporta mayor concentración de carbohidratos, al representar el $46 \%$ de la biomasa.

Con el fin de tener un panorama general, en la Fig.1 se muestra el comportamiento desde el primer hasta el último día de experimentación para la producción porcentual de carbohidratos, a partir del consumo de la fuente de nitrógeno, fósforo y carbono (glicerina) en su mejor tratamiento. Se observa entonces una acumulación de los mismos a partir del día 4; cuando se hace presente la limitación o consumo de nutrientes como fósforo y nitrógeno.

\subsection{Producción de proteínas}

La mayor concentración de proteínas se obtuvo en el tratamiento 9, con un valor de $4.32 \mathrm{~g} / \mathrm{L}$ que corresponde al $20 \%$ de la biomasa. Este tratamiento contó con una concentración inicial de glicerina $5 \% \mathrm{v} / \mathrm{v}$ y una concentración inicial de nitrógeno $0.37 \mathrm{~g} / \mathrm{L}$.
De la misma manera fue imperativo ver el cambio porcentual en la producción de proteínas respecto a la biomasa, a través del consumo de nutrientes y sustrato con el paso de los días experimentales, Fig. 2. Hasta el día 8 existe un aumento de proteínas. En días posteriores se registra un descenso de este metabolito debido a la limitación de nutrientes.

\subsection{Producción de lípidos}

En la producción de lípidos no se encontró día o tratamiento significativo, pero se evidencia, a través del análisis de datos, que hubo una producción superior de estos durante todos los días del tratamiento 15, Tabla III, el cual presentaba una concentración baja de nitrógeno $0.04 \mathrm{~g} / \mathrm{L}$ y una concentración media de glicerina $3 \% \mathrm{v} / \mathrm{v}$.

Para este metabolito la limitación en los nutrientes es de suma importancia, por lo que se estudió el comportamiento de la producción de lípidos a partir del consumo de nitrógeno, fósforo y glicerina durante el tiempo experimental. Se puede observar en la Fig. 3, que la producción de lípidos se favorece después del día 12, al encontrarse la microalga frente a una limitación de nutrientes.

TABLA II

COMPARACIÓN MEJORES TRATAMIENTOS DÍA 12 PARA CARBOHIDRATOS

\begin{tabular}{|c|c|c|c|c|}
\hline Tratamientos & Glicerina $(\% \mathrm{v} / \mathrm{v})$ & Fuente de Nitrógeno $(\mathrm{g} / \mathrm{L})$ & Carbohidratos $(\mathrm{g} / \mathrm{L})$ & Biomasa $(\mathrm{g} / \mathrm{L})$ \\
\hline 1 & 1 & 0.12 & $1.78 \pm 0.05$ & $9.81 \pm 0.05$ \\
\hline 3 & 5 & 0.37 & $5.34 \pm 0.05$ & $11.62 \pm 0.05$ \\
\hline
\end{tabular}

Fuente: los autores.

Fig. 1. CONSUMO FUENTE DE NITRÓGENO A), FÓSFORO B) Y GLICERINA C) VS PRODUCCIÓN CARBOHIDRATOS [\%], TRATAMIENTO 3

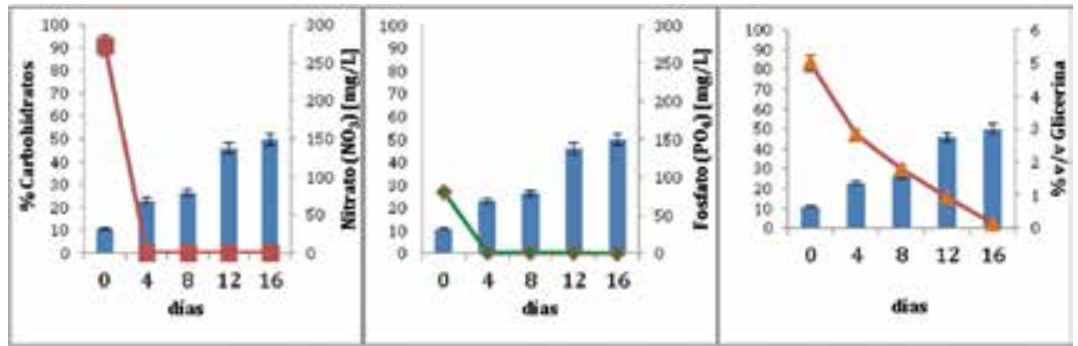

- Carbohidratos = Nitrato Eosfato = Glicerol

Fuente: los autores. 
Fig. 2. CONSUMO FUENTE DE NITRÓGENO A), FÓSFORO B) Y GLICERINA C) VS PRODUCCIÓN PROTEÍNAS [\%], TRATAMIENTO 9

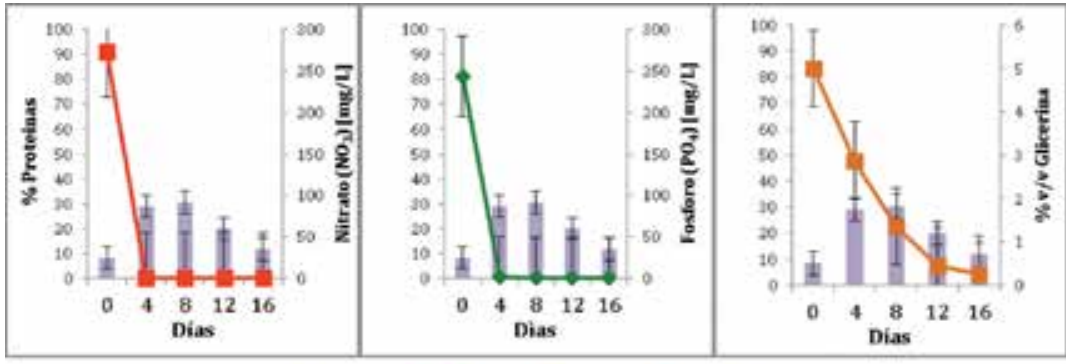

NProteinas = Nitrato $=$ fosfato $=$ Glicerol

Fuente: los autores.

TABLA III

PRODUCCIÓN PORCENTUAL LÍPIDOS RESPECTO A LA PRODUCCIÓN DE BIOMASA (g/L) MEJOR TRATAMIENTO

\begin{tabular}{|c|c|c|c|c|c|}
\hline Tiempo (día) & 0 & 4 & 8 & 12 & 16 \\
\hline Biomasa (g/L) & $14.13 \pm 0.05$ & $30.03 \pm 0.05$ & $22.73 \pm 0.05$ & $24.07 \pm 0.05$ & $20.23 \pm 0.05$ \\
\hline Lípidos \% & 15 & 9 & 11 & 15 & 11 \\
\hline
\end{tabular}

Fuente: Ios autores.

Fig. 3. CONSUMO FUENTE DE NITRÓGENO A), FÓSFORO B) Y GLICERINA C) VS PRODUCCIÓN LÍPIDOS [\%], TRATAMIENTO 150

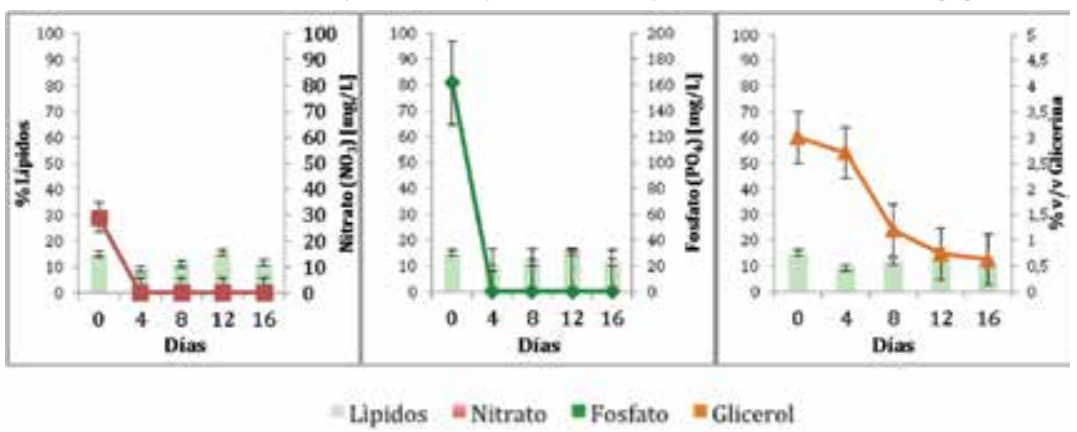

Fuente: los autores

\section{ANÁLISIS DE RESULTADOS}

La cantidad de carbono fijado para producción de carbohidratos, proteínas y lípidos se ve muy influenciada por la disponibilidad de nutrientes. Es por esto que las microalgas al ser expuestas a diversas condiciones permiten modificaciones en la producción de biomasa y metabolitos presentes en su composición celular. Existen dos macronutrientes importantes en el crecimiento y el metabolismo de las células, que son el nitrógeno y fósforo, los cuales son parte fundamental para la formación de proteínas y ácidos nucleicos, además de estar incluidos en la columna vertebral del ADN y ARN como moléculas esenciales en las células vivas. Se pudo evidenciar que, una limitación severa en dichos nutrientes, aumenta la acumulación de lípidos y carbohidratos, lo que concuerda con Brányiková et al. [19], y Belotti et al. [20], confirmando que bajo condiciones de estrés, en este caso de limitación de nutrientes, se generó un aumento de metabolitos de interés; sin embargo, analizando el efecto de la limitación de nutrientes en la producción de proteínas, ocurre totalmente lo contrario, ya que para que exista un aumento de proteínas debe ir acompañado de un mayor contenido de nutrientes, confirmando lo reportado en la literatura según Shifrin et al. [21].

Respecto a la glicerina crudo, cabe resaltar que fue consumido totalmente por la microalga $C$. 
vulgaris en todos los tratamientos. De la misma manera, se prueba que la inmovilización constituye una parte fundamental en la adaptación de la microalga a este sustrato, además de impedir posibles procesos de inhibición [22], facilitando adicionalmente la extracción de la biomasa.

Al realizar una comparación con los resultados obtenidos por Estevez et al. [23], este estudio encontró una concentración superior respecto a la producción de biomasa y lípidos bajo una concentración de 3\% de glicerol y cantidades similares de nitrógeno. Vale la pena resaltar que el glicerol utilizado en este estudio no tuvo tratamiento previo, debido a los costos que el proceso genera. Los resultados obtenidos basados en la concentración de biomasa son positivos, teniendo en cuenta que la baja concentración de biomasa ha sido obstáculo en los cultivos microalgales para que el proceso sea comercialmente asequible, considerando el costo de recolección y su separación [24], lo que demuestra la viabilidad de usar glicerol sin tratamiento previo como fuente de carbono.

El alto contenido de lípidos hace atractiva a las microalgas para la producción de biocombustibles, según Hu et al. [7]. Sin embargo, es posible aprovechar los demás metabolitos presentes en la biomasa, tal es el caso del uso de estas como fuente de proteínas, para la producción de alimentos de alto valor nutricional [25], teniendo en cuenta que además del consumo de glicerina por la microalga durante el tiempo de cultivo, se pudo observar una alta producción de los diferentes metabolitos, Tabla IV, aún para el tratamiento donde se obtuvieron altos valores de lípidos. Cabe resaltar que aunque se obtuvieron producciones similares de biomasa en los mejores tratamientos y aún en el tratamiento control, existe una notable diferencia en la producción de metabolitos, obteniéndose una cantidad muy superior en cultivos con glicerina en comparación con el cultivo control.

\section{CONCLUSIONES}

De acuerdo con los resultados obtenidos en la experimentación se puede decir que la glicerina constituye una fuente de carbono potencial para la Chlorella vulgaris UTEX 1803, encontrándose como concentraciones óptimas un rango entre $3 \mathrm{y}$ $5 \% \mathrm{v} / \mathrm{v}$.

Para estimular la acumulación de lípidos y carbohidratos debe existir una limitación en las fuentes de nitrógeno y fósforo. Sin embargo, para favorecer la acumulación de proteínas se obtuvo que el proceso se comporta de forma totalmente opuesta.

La utilización de glicerina como sustrato para el cultivo de las microalgas constituye una alternativa promisoria, debido a que ayuda a mitigar el impacto ambiental del proceso de producción de biodiesel asociado con la disponibilidad de glicerina y ofrece una diversificación en la industria de los biocombustibles bajo el concepto de biorrefinería.

TABLA IV

RESUMEN MEJORES TRATAMIENTOS PRODUCCIÓN CARBOHIDRATOS, PROTEÍNAS Y LÍPIDOS, PARA EL MEJOR DÍA DE EXPERIMENTACIÓN (DÍA 12)

\begin{tabular}{|c|c|c|c|c|c|}
\hline \multicolumn{2}{|r|}{ Tratamiento } & Biomasa (g/L) & Proteínas \% & Carbohidratos \% & Lípidos \% \\
\hline \multirow{2}{*}{ T9 } & 5\% Glicerina & \multirow{2}{*}{$21 \pm 0.05$} & \multirow{2}{*}{20} & \multirow{2}{*}{14} & \multirow{2}{*}{0.015} \\
\hline & $0.37 \mathrm{~g} / \mathrm{L} \mathrm{P}-0.37 \mathrm{~g} / \mathrm{L} \mathrm{N}$ & & & & \\
\hline \multirow{2}{*}{ T15 } & 3\% Glicerina & \multirow{2}{*}{$14 \pm 0.05$} & \multirow{2}{*}{15} & \multirow{2}{*}{29} & \multirow{2}{*}{15.5} \\
\hline & $0.25 \mathrm{~g} / \mathrm{LP}-0.04 \mathrm{~g} / \mathrm{L} \mathrm{N}$ & & & & \\
\hline \multirow{2}{*}{ T3 } & 5\% Glicerina & \multirow{2}{*}{$12 \pm 0.05$} & \multirow{2}{*}{22} & \multirow{2}{*}{46} & \multirow{2}{*}{0.025} \\
\hline & $0.12 \mathrm{~g} / \mathrm{L} \mathrm{P}-0.37 \mathrm{~g} / \mathrm{L} \mathrm{N}$ & & & & \\
\hline \multirow{2}{*}{ Control } & 0\% Glicerina & \multirow{2}{*}{$25 \pm 0.05$} & \multirow{2}{*}{3} & \multirow{2}{*}{6} & \multirow{2}{*}{0.011} \\
\hline & $0.25 \mathrm{~g} / \mathrm{LP}-0.25 \mathrm{~g} / \mathrm{L} \mathrm{N}$ & & & & \\
\hline
\end{tabular}

Fuente: los autores.

\section{REFERENCIAS}

[1] P. Spolaore, C. Joannis-Cassan, E. Durán, \& A. Isambert, "Commercial applications of microalgae". Journal of bioscience and bioengineering, 101(2), pp. 8796, 2006.
[2] J. V. Haveren, E. L. Scott, and J. Sanders, "Bulk chemicals from biomass". Biofuels, Bioproductos \& Biorefining. vol 2, pp. 41-57, 2008.

[3] L. Christenson, \& R. Sims, "Production and harvesting of microalgae for wastewater treatment, biofuels, and 
bioproducts". Biotechnology Advances, vol. 29, No. 6 . pp. 686-702, 2011.

[4] N. Shifrin, \& S. Chisholm, "Phytoplankton Lipids: Interspecific Differences and Effects of Nitrate, Silicate and Lightdark Cycles". J. Phycol., vol 17, pp. 372-384, 1981.

[5] O. Zacheus, E. livanainen, T. Nissinen, M. Lehtola, \& P. Martikainen, "Bacterial Biofilm Formation on Polyvinylchloride, Polyethylene And Stain Less Steel Exposed Too Zonated Water". Water Res., vol. 34. pp. 63-70, 2000.

[6] E. Leenen, V. Dos Santos, K. Grolle, J. Tramper, R. Wijffels, "Characteristics of and selection criteria for support materials for cell immobilization in wastewater treatment”. Water Res., 12, pp. 2985-2996, 1996.

[7] Q. Hu, M. Sommerfeld, E. Jarvis, M. Ghirardi, M. Posewitz, M. Seibert, A. Darzins, "Microalgal triacylglycerols as feedstocks for biofuel production: Perspectives and advaces". Plant J., 54, pp. 621-639, 2008.

[8] C. Zamalloa, N. Boon, \& W. Verstraete, "Decentralized two-stage sewage treatment by chemical-biological flocculation combined with microalgae biofilm for nutrient immobilization in a roof installed parallel plate reactor". Bioresource technology, 130, pp. 152-160, 2013.

[9] C. H. Hsieh, \& W. T. Wu, "Cultivation of microalgae for oil production with a cultivation strategy of urea limitation". Bioresource technology, 100(17), pp. 39213926, 2009.

[10] N. Patil, Y. Veeranagouda, M. Vljaykumar, S. Nayak, T. Karegoudar, "Enhanced and potential degradation of o-phthalate by Bacillus sp. Immobilized cells in alginate and polyurethane". International Journal of Biodetergents Biodegradable, 57, pp. 82-87, 1996.

[11] Y. Liang, N. Sarkany, Y. Cui, "Biomass and lipid productivities of Chlorella vulgaris under autotrophic, heterotrophic and mixotrophic growth conditions". Biotechnol Lett., 31, pp. 1043-1049, 2009.

[12] C. I. Domínguez, Z. Arbib, F. Chinalia, S. C. Oliveira, J. Perales, P. Almeida, J. I. Druzian, I. Andrade, "From waste to energy: Microalgae production in wastewater and glycerol". Applied Energy, 109, pp. 283-290, 2013.

[13] L. S. Clesceri, A. E. Greenberg, A. D. Eaton, Standard methods for examination of water and wastewater. American Public Health Association, 20th ed. Washington, D.C., 1999.

[14] M. Dubois, K. Gilles, P. Rebers, F. Smith. "Colorimetric Method for Determination of Sugars and Related Substances". Analytical Chemistry, 3(28), pp. 350356,1956
[15] Y. Chen, S. Vaidyanathan, "A simple, reproducible and sensitive spectrophotometric method to estimate microalgal lipids". Analytica Chimica Acta, 724, pp. 67 72,2012

[16] Y. Chen, \& S. Vaidyanathan, "Simultaneous assay of pigments, carbohydrates, proteins and lipids in microalgae". Analytica chimica acta, 776, pp. 31-40, 2013.

[17] O. H. Lowry, N. J. Rosenbrough, A. L. Farr, R. J. Randall, "Protein measurement with the Folin Phenol Reagent". Journ. Biol. Chem, 193, pp. 65-275, 1951.

[18] C. R. Dorey, \& J. A. Draves. Quantitative analysis laboratory: a new approach. Laboratory Manual. Department of Chemistry, University of Central Arkansas, Conway, AR. 1998.

[19] I. Brányiková, B. Maršálková, J. Doucha, T. Brányik, K. Bišová, V. Zachleder, M.Vítová, "Microalgae novel highly efficient starch producers". Biotechnology Bioengineering, 108 (4), pp. 766-776, 2011

[20] G. Belotti, M. Bravi, B. De Caprariis, P. De Filippis, M. Scarsella, "Effect of Nitrogen and Phosphorus Starvations on Chlorella vulgaris Lipids Productivity and Quality under Different Trophic Regimens for Biodiesel Production". American Journal of Plant Sciences, 4, pp. 44-51, 2013.

[21] [21] N. S. Shifrin, S. W. Chisholm, "Phytoplankton lipids: Interspecific differences and effects of nitrate, silicate and lightdark cycles". J. Phycol., 17, pp. 372384, 1981.

[22] S. Silveira Martins, C. Martins, L. Cidrao, S. Santaella, "Immobilization of microbialcells: A promising tool for treatment of toxic pollutants in industrial wastewater". African Journal of Biotechnology, 12 (28), pp. 44124418, 2013.

[23] L. Estévez, A. Barajas, C. Barajas, V. Kafarov, “Improvement of Lipid Productivity on Chlorella vulgaris Using Waste Glycerol and Sodium Acetate". Ciencia, Tecnología y Futuro, 5 (2), pp. 113-126, 2013.

[24] T. Heredia-Arroyo, W. Wei, R. Ruan, \& B. Hu, "Mixotrophic cultivation of Chlorella vulgaris and its potential application for the oil accumulation from non-sugar materials". Biomass and Bioenergy, 35 (5), pp. 22452253, 2011.

[25] E. W. Becker, "Microalgae as a source of protein". Biotechnological Advances, 25, pp. 207-210, 2007. 